\title{
ALGUNOS ASPECTOS DE LOS MEDIOS ENTONATIVOS EN ESPAÑOL
}

0. Antes de comenzar mis explicaciones, quisiera dar a conocer los puntos de partida de mis análisis. En primer lugar, he de señalar que los métodos de mi trabajo se han visto influidos por el hecho de enseñar a los futuros traductores e intérpretes, en cuya carrera uno de los aspectos fundamentales es la constante comparación del español con el checo ( $\mathrm{y} / \mathrm{o}$ el eslovaco). La concepción comparativa de los análisis trae consigo tanto ventajas como desventajas, ya que ver los fenómenos de un idioma a través de las categorías de otro permite, a veces, abrir nuevos enfoques del problema, pero, por otra parte, un extranjero nunca puede percibir un idioma como si fuese su hablante nativo.

Para analizar la entonación en un idioma determinado podemos partir de dos niveles diferentes:

1. Tomar en cuenta el aspecto físico del contorno melódico.

2. Analizar su aspecto funcional, es decir, la posición que ocupan los fenómenos entonativos (ante todo melódicos) entre los demás medios lingüísticos que sirven en una lengua determinada para delimitar, diferenciar e integrar contextualmente una oración.

1. Desde el punto de vista del signo lingüístico, podemos decir que el aspecto físico de la curva melódica es el nivel de puro "significante", lo que no representa un punto de partida suficiente para los análisis lingüísticos de la entonación en toda su complejidad (Romportr, 1973, pág. 185). Abstra- 
yendo en lenguas diferentes los análisis de los contornos melódicos de las oraciones, de su función, se llegó a la conclusión de que aquéllos presentan ciertas afinidades: los de las preguntas pronominales y de las oraciones afirmativas son descendentes, los de las preguntas absolutas, ascendentes. Sin embargo, en algunas obras (p. ej., Guiraud, 1974, pág. 66) suele hablarse del carácter paralingüístico de la entonación (mejor dicho, melodía oracional).

Al analizar desde el punto de vista mencionado las concepciones de la entonación española, vemos que hay dos actitudes ante la interpretación del contorno melódico de sus oraciones.

En la primera concepción (Navarro, $1966^{1}$; Matluck, 1965) se considera que las diferencias entre los contornos melódicos de la oración afirmativa y de la interrogativa residen también en su inflexión inicial, aun siendo fonológicamente relevante ante todo su inflexión final. Esta concepción se apoya, hasta cierto punto, también en la ortografía española : al principio de la oración interrogativa se escribe el signo de interrogación, al principio de la exclamativa, el signo de admiración (Gili GAYA, 1970, página 46).

Si nos planteamos el problema del supuesto carácter discontinuo de la forma melódica relevante en español y tratamos de aplicar dicha concepción a un análisis concreto del material lingüístico, llegamos a la conclusión de que aquélla no está bien justificada. Al someter a un análisis musical varias oraciones españolas, llegamos a la conclusión de que no puede haber diferencias fundamentales entre la inflexión inicial (el intervalo musical entre la sílaba inicial absoluta y la primera sílaba acentuada) de una oración interrogativa y la de una oración afirmativa.

Por lo tanto, somos partidarios de la otra concepción (p. ej., Cárdenas, 1960 ; Dalbor, 1969; Quilis, Fernández, 1973; Quilis, 1981), según la cual resulta fonológicamente relevante sólo la inflexión final de la oración (resp. del grupo fónico-Quilis, Fernández, 1973). Esta concepción se basa también en el hecho de que la melodía oracional no debe considerarse como una suma de alturas tonales absolutas, sino como un continuo contorno melódico, en el cual es necesario considerar la altura tonal de sus componentes como relativa dentro de ese contorno (en lugar de "altura tonal" nos parece más justificado hablar de descenso o ascenso total). Cuestionando desde este punto de vista la relevancia distinta de la inflexión inicial, vemos que a las sílabas iniciales de la oración les falta ese contacto con las sílabas antecedentes, $y$, por lo tanto, no responden al mencionado criterio de la continuidad del contorno melódico de la oración.

Tomar como punto de partida del análisis de la entonación la inflexión 
final del grupo fónico (Quilis, Fernández, 1973) permite, como es sabido, diferenciar las oraciones (enunciados) completas, terminadas en cadencia 0 anticadencia de las incompletas, terminadas en suspensión, semicadencia y/o semianticadencia. Considerar fonológicamente relevante la inflexión final de la oración (del enunciado -CÁRDEnAs, 1960; DALBOR, 1969) permite hacer la distinción fundamental entre las oraciones afirmativas y las interrogativas. Definiendo el contorno melódico de la inflexión final, tanto del grupo fónico como de la oración (enunciado), aquél se considera delimitado, como es sabido, por la última sílaba acentuada de la respectiva unidad. En este aspecto de la definición de la forma relevante del contorno melódico en español, ya se nota una estrecha interrelación de sus prosodemas, destacándose, naturalmente, sus componentes melódicos. Por lo tanto, somos de la opinión de que para denominar la forma melódica relevante en español, el término más apropiado es el de melodema.

Las variantes del melodema se deben, en primer lugar, a las diferencias del número de sílabas que lo forman. Analizando desde este punto de vista las posibles variantes del melodema (para simplificar, en la oración formada por un solo grupo fónico), vemos que puede haber las variantes siguientes:

A.

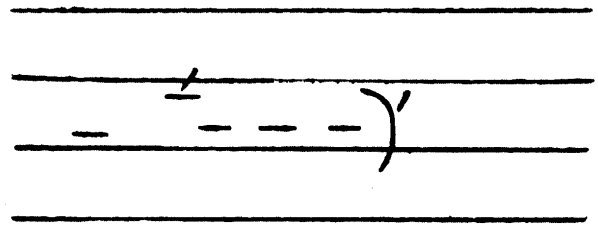

Termina de comer.

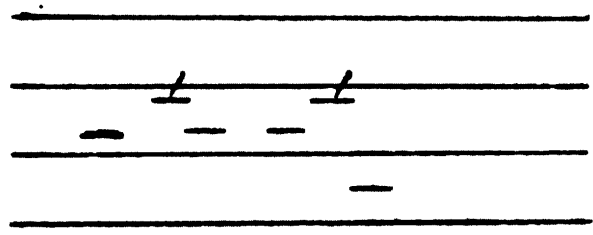

Termina cansado.

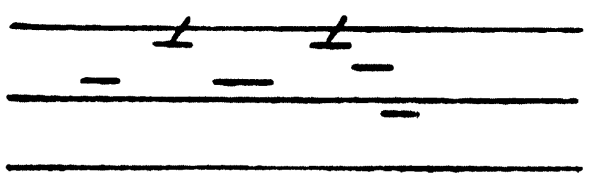

Termina el sábado.
B.

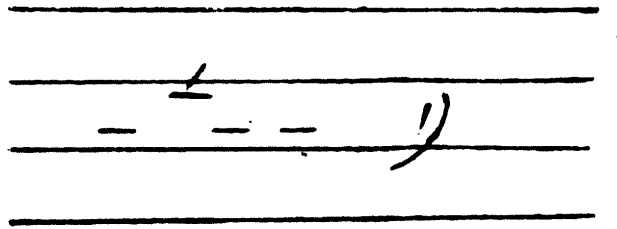

¿Termina de comer?

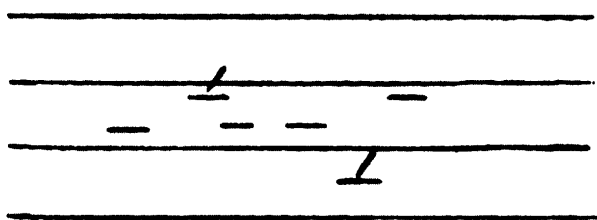

\section{¿Termina cansado?}

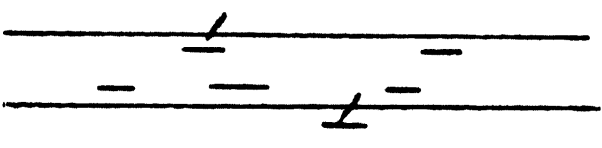

¿Termina el sábado? 


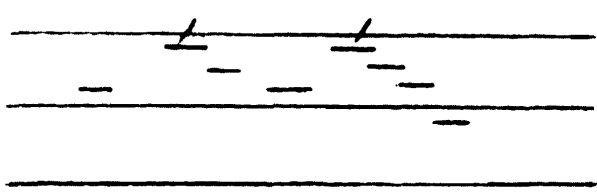

Termina diciéndoselo.

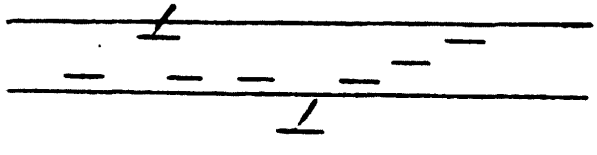

¿Termina diciéndoselo?

Para estas variaciones de la forma melódica relevante adoptamos el término de alómelo (Rom PORTL, 1971, 1981), comprendido como fenómeno análogo a la variante posicional de los fonemas; está dado por el número de sílabas postónicas que lo forman.

2. Sin embargo, como ya se ha podido ver en la delimitación del melodema en español, los componentes de la melodía oracional están estrechamente relacionados con otros fenómenos prosódicos del idioma determinado (p. ej., Romporte, 1970, 1971; Antipova, 1979). Por lo tanto, compartimos la concepción (RomportL, 1970, págs. 9-18; 1973, págs. 131-185) de que hace falta diferenciar entre sí los antes mencionados conceptos de contorno melódico y melodema, por una parte, y los de entonación y entonema, por otra. Los primeros se refieren, como ya se ha dicho anteriormente, a los fenómenos puramente melódicos, mientras que los segundos los consideramos como fenómenos complejos, compuestos de elementos musicales, cuantitativos y de intensidad.

Esta diferenciación justifica también la existencia de la entonación como fenómeno lingüístico: tiene carácter sistemático, el repertorio y estructuración de sus posibles componentes varían de una lengua a otra, así como su relación con los demás medios lingüísticos que en la lengua dada sirven para delimitar y diferenciar la oración (enunciado - RomporTL, 1970, página 210). Como ejemplo, podemos señalar la comparación de dichos fenómenos lingüísticos en español y en checo.

Comparando el repertorio de los medios suprasegmentales en checo y en español, las diferencias más notables entre los dos idiomas aparecen en el campo del aprovechamiento del acento y de la cantidad vocálica. Mientras que en checo, la cantidad vocálica es fonológicamente relevante, es decir, existen las parejas mínimas diferenciadas por la cantidad (duración) como /rada/ consejo y /rāda/ encantada; y el acento tiene la función de la señal demarcativa, en español la situación es diferente: el acento es, como se sabe, fonológico (célebre - celebre - celebré) y los cambios de duración se consideran más bien como emocionales (p. ej., Martinet, 1972). Sin embargo, la concepción de la duración (cantidad vocálica) en español como 
puramente emocional, nos parece un poco simplificada: en dicha lengua existen relaciones entre la posición del acento, el tipo de sílaba (libre o trabada) y su duración (NAVARRo, 1966², págs. 197-207).

La presencia lingüística de ciertas cualidades prosódicas o su ausencia en diferentes idiomas sirvió de base para la tipología entonativa (ROMPORTL, 1973, págs. 131-136). De este modo, los idiomas se dividen en cuatro tipos entonativos.

En el primero, entran las lenguas cuya característica entonativa es una cualidad silábica y oscila dentro de sus límites. En ellas, la sílaba es generalmente idéntica al semantema. Es el caso de las llamadas lenguas tonales.

En las lenguas del segundo tipo. se caracterizan entonativanente unidades semánticas superiores (las palabras), que pueden tener una o más sílabas. Se trata de lenguas que poseen el llamado "acento melódico"; es decir, las diferencias entonativas están ligadas a diferencias entre la sílaba acentuada y la inacentuada. Al mismo tiempo, los medios entonativos pueden aprovecharse también en el nivel de la fonología oracional.

En los idiomas del tercer tipo, los medios entonativos son funcionales en el nivel de la oración, destacándose entre ellos, ante todo, el contorno melódico; los componentes dinámicos, temporales y el timbre quedan aparte. Dentro de este tipo entonativo se incluyen no sólo los idiomas, en los cuales existe la cantidad vocálica (el checo), sino también los que poseen calidad silábica (el ruso) y los que aprovechan el llamado "acento dinámico" en la fonología de la palabra.

El cuarto tipo abarca los idiomas en los cuales la entonación no se aprovecha lingüísticamente.

Considerando la concepción tradicional de los medios prosódicos en español, podríamos decir que los dos idiomas que se encuentran en el centro de nuestra atención (el checo y el español) pertenecen al tercer tipo entonativo. Sin embargo, tomando en cuenta los resultados de los análisis acústicos del acento español, la situación llega a ser diferente.

Partiendo de los análisis instrumentales del acento español (QurLIs, 1971, 1981), se confirmaron las hipótesis anteriores de que en la percepción del acento español no desempeña un papel primordial la intensidad, sino la frecuencia del fundamental. Sobre la base de estas afirmaciones podemos llegar a la conclusión de que el español pertenece al segundo tipo entonativo, es decir, aprovecha los medios entonativos (melódicos) tanto en la fonología de la palabra como en la oracional.

La determinación del tipo entonativo del idioma abre, al mismo tiempo, las puertas para los análisis del segundo conjunto de problemas: los relacionados con el aspecto funcional de la entonación oracional. Sin embargo, 
aun en los idiomas pertenecientes a un mismo tipo entonativo, estos medios pueden ocupar un lugar diferente en la jerarquía de todos los medios lingüísticos, que en el idioma determinado sirven para delimitar, definir e integrar contextualmente una oración (un enunciado) -en adelante vamos a emplear en este sentido el término de jerarquía comunicativa.

Dentro de esta jerarquía comunicativa entran tanto los medios fonéticos (p. ej., la localización del acento dinámico, las pausas, etc.) como los medios de otros niveles lingüísticos (la existencia o ausencia de palabras interrogativas especiales, las reglas del orden de los elementos, etc.) en un idioma determinado (Romports, 1971, pág. 210). Quisiéramos todavía agregar que los aspectos mencionados de los medios entonativos no se pueden analizar separados, sino en toda su complejidad.

3. Analizando desde estos puntos de vista la entonación española, podemos llegar a conclusiones que discrepen notablemente de las concepciones que hemos tenido a nuestro alcance. En este lugar, y antes de seguir adelante, quisiéramos reiterar dos observaciones: Nuestra concepción del español naturalmente ha sido y sigue siendo influida por nuestra condición de extranjera. Por lo tanto, nuestra concepción de dicho problema ha sido siempre, aunque implícitamente, comparativa; es decir, la entonación española se ha concebido y analizado bajo el prisma de una lengua diferente.

Sin embargo, no aspiramos a que nuestras conclusiones tengan un valor universal: nuestro objetivo es sólo el intento de descubrir algunas características poco conocidas de los fenómenos entonativos del español.

Del concepto del tipo entonativo se desprende, pues, lo siguiente: Si las lenguas de un mismo tipo pueden ofrecer diferencias notables en la posición de los medios entonativos en la jerarquía comunicativa, estas diferencias se destacan aún más en las lenguas de diferentes tipos entonativos. En este lugar cabe preguntarse: ¿cuál es la posición de la entonación en la jerarquía comunicativa en español y cuáles son los demás medios lingüísticos que influyen en ella?

Somos de la opinión de que la posición de un fenómeno en la jerarquía comunicativa en una lengua determinada está estrechamente relacionada con el repertorio de los medios de integración contextual existentes en dicha lengua. Dentro del repertorio de dichos medios entrarán en español tanto los medios fónicos, ante todo prosódicos, que son el centro de nuestro interés, como por ejemplo el artículo, el orden de los elementos (Tichí, 1961), o, mejor dicho, las reglas que lo rigen (DANeš, 1967, pág. 509). En resumen, podríamos decir que el papel de los medios prosódicos (ante todo el de la entonación) en la integración contextual de los elementos se 
reduce conforme aumente la importancia de los medios de los demás niveles lingüísticos. Por lo tanto, en un idioma que no posea la categoría del artículo (como el checo), la posición de la entonación entre los medios de integración contextual es totalmente diferente de la que se produce en los idiomas que la poseen. Asimismo, la variabilidad del orden de los elementos influye sobre la relativa uniformidad de la posición del entonema y viceversa.

Hemos tratado de resumir los problemas fundamentales que se presentan al tratar de analizar la posición de la entonación española en la jerarquía comunicativa de dicho idioma. Ahora quisiéramos esbozar cómo influyen las características mencionadas sobre la interpretación del contorno melódico de las oraciones españolas.

En nuestros análisis hemos introducido el concepto del llamado centro enunciativo de la oración y lo hemos identificado con la palabra proeminente de la oración, entendiendo la proeminencia (el "acento oracional") como un complejo de elementos de intensidad, cantidad y entonación (cf. Hocketr, 1972, pág. 46). Dicho de otra manera: la sílaba acentuada de la palabra proeminente se destaca no sólo por su intensidad, sino también por su duración y la identificación con el centro (la culminación) entonativo de la oración. A partir de esta sílaba empieza a realizarse el melodema de la oración correspondiente. Las hipótesis mencionadas nos sirvieron como base para la siguiente interpretación del contorno melódico de las oraciones hispánicas.

\subsection{Oraciones afirmativas.}

$\mathrm{Si}$ analizamos los contornos melódicos de las oraciones modelo antes mencionadas (págs. 4-5), nos damos cuenta de que la última sílaba acentuada en nuestros casos coincide con la acentuada de la palabra proeminente de la oración, lo que tiene por consecuencia un marcado ascenso tonal en comparación con las sílabas anteriores, y después de ella, se realiza el descenso melódico correspondiente al melodrama descendente de la oración afirmativa (A.).

Sin embargo, al analizar oraciones de una estructura sintáctica más complicada, la proeminencia puede caer en otro segmento, no en la última palabra : 
C.

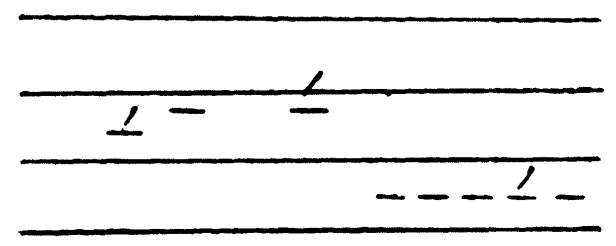

Bueno ..., muy interesante.

Oraciones como ésta justifican la introducción de los conceptos como centro enunciativo y centro entonativo para delimitar el entonema en español: en su esquema de entonación se nota que el melodema descendente comienza con la sílaba acentuada correspondiente a la palabra "muy".

Por otra parte, tomando en cuenta, en la definición del melodema en español, el centro entonativo y no, mecánicamente, la última sílaba acentuada, aumenta naturalmente el número de sílabas que pueden formar el melodema, y, por consiguiente, el número de posibles variaciones del contorno melódico: de los alómelos, comprendidos, como ya se dijo antes, como variantes posicionales del melodema, lo que puede permitir, a nuestro parecer, profundizar también las investigaciones dialectológicas. Hemos tratado de aplicar nuestros criterios en los análisis de la llamada "entonación circunfleja" en el español mejicano (cf. Matruck, 1966, págs. 30 y sigs.). Si comparamos los esquemas de la oración "Están contentos", pronunciada:

D. con la entonación culta (mejor dicho, indiferente):

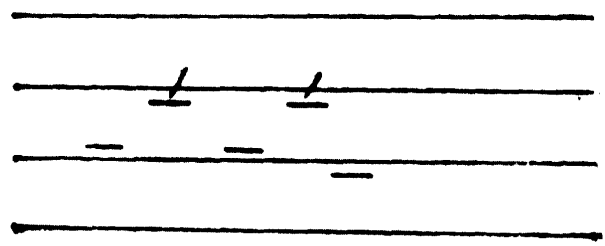

Están contentos.

E. con la "entonación circunfleja":

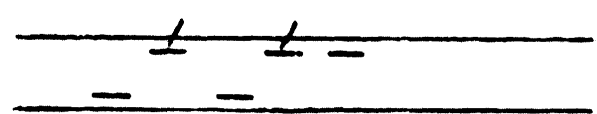

Están contentos. 
vemos, entre otras cosas, que el ascenso melódico de la sílaba acentuada de la palabra proeminente representa el fenómeno relevante para determinar el valor afirmativo de la oración: después sigue el melodema descendente (entonación culta) o el horizontal ("entonación circunfleja”).

\subsection{Oraciones interrogativas.}

En los análisis de las oraciones interrogativas vamos a devidirlas en tres grupos :

1. Preguntas pronominales.

2. Preguntas absolutas.

3." "Metapreguntas".

3.2.1. Preguntas pronominales. Bajo este concepto comprendemos las oraciones interrogativas que comienzan por un pronombre, adjetivo, adverbio $\mathrm{u}$ otra partícula interrogativa, como por ejemplo:

F.

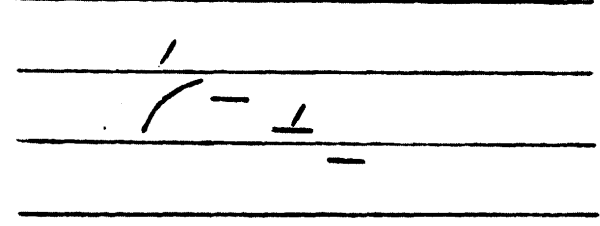

¿Dónde fuiste? Cf. Quilis, Fernández, 1973, págs. 176 y sigs.

Estas preguntas, así como las antes mencionadas oraciones afirmativas, también ofrecen un melodema descendente. Este melodema descendente comienza, generalmente, a partir de la sílaba acentuada del elemento interrogativo, el cual se convierte en el fenómeno proeminente de la oración. El elemento interrogativo representa, en nuestra concepción, el centro enunciativo, y, por consiguiente, el centro entonativo de la oración.

En este fenómeno parecen basarse también las concepciones del carácter discontinuo de la forma melódica relevante en español (NAVARRo, 1966 ${ }^{\mathbf{~}}$; Matluck, 1965 ; Gili Gaya, 1970). Encontrándose el centro entonativo del melodema descendente al principio de la pregunta pronominal, es natural que las sílabas iniciales puedan pronunciarse a una altura tonal más elevada, comparadas con las sílabas iniciales de la oración afirmativa que no forman 
parte del contorno melódico relevante formado también por el melodema descendente.

En este lugar cabe preguntarse cuántos melodemas descendentes existen en español. Ya que, a primera vista, en el campo del contorno melódico no aparecen diferencias notables, las oraciones afirmativas, así como las preguntas pronominales, presentan un descenso tonal de las sílabas postónicas (mejor dicho, postproeminentes).

Sin embargo, como dijimos antes, en el análisis de los medios melódicos no podemos hacer caso omiso de sus relaciones con los elementos de los demás niveles lingüísticos, y aquí sí vemos una diferencia notable: En la pregunta pronominal, la proeminencia está ligada al interrogativo, identificado con la culminación enunciativa y entonativa, mientras que las reglas de identificación de la culminación enunciativa en una oración afirmativa son más complicadas y se relacionan con toda una serie de recursos de integración contextual de la oración en el idioma en cuestión (Tichý, 1961, pág. 97 ; Daneš, 1967. pág. 509).

3.2.2. Preguntas absolutas. Se diferencian de las oraciones afirmativas ante todo por su contorno melódico, aunque no se puede negar la importancia de la inversión de los elementos para convertir una frase afirmativa en la interrogativa (cf. Quilis, Fernández, 1973, págs. 174-175).

Primero vamos a dedicar nuestra atención al contorno melódico de las preguntas absolutas en el esquema B (págs. 4-5). Al analizarlas desde nuestro punto de vista, vemos que el melodema ascendente comienza a partir de la útima sílaba acentuada de la oración, la cual, en nuestros casos, coincide con la sílaba acentuada de la palabra proeminente. El número de sílabas que la siguen influye después en la realización del melodema ascendente y da origen a cierto número de alómelos como variantes posicionales de aquél.

Sin embargo, ahora cabe analizar otro aspecto del contorno melódico de las preguntas absolutas en español, ya que, como se dijo antes, éstas pueden formarse por medio de dos procedimientos lingüísticos: por la entonación (véase más arriba) y por la inversión de los elementos. Los dos procedimientos representan medios de la integración contextual en español ; por lo tanto, trataremos de ver la influencia de la coincidencia de ambos sobre el contorno melódico de las preguntas absolutas en español.

Al finalizar el contorno melódico de una pregunta absoluta, hecha por la inversión de los elementos (Quilis, Fernández, 1973, pág. 175): 
G.

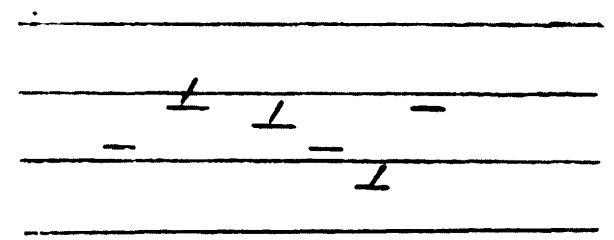

¿Es tuyo este libro?

nos damos cuenta de que, en la oración mencionada, parece haber dos núcleos melódicos: Uno de la sílaba acentuada de la palabra tuyo y el otro en la acentuada de libro. Al contrario, sin la inversión de los elementos, el contorno melódico sería distinto (en una enunciación no enfática) :

$\mathrm{H}$.

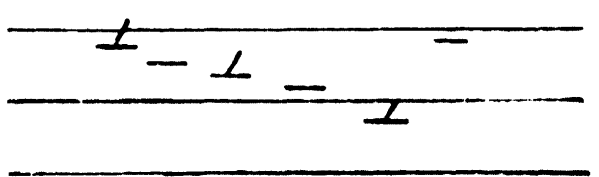

¿ste libro es tuyo?

Las diferencias en los contornos melódicos de las dos oraciones parecen encontrarse en la parte anterior a la línea tonal relevante, definida tradicionalmente como la parte del contorno melódico a partir de la última sílaba acentuada.

En la primera ocasión mencionada, con la inversión de los elementos, se produce primero un ascenso melódico que llega hasta la primera sílaba acentuada; sigue un descenso tonal, hasta la última sílaba acentuada, después de la cual aparece un "normal" contorno melódico ascendente. Sin embargo, en la pregunta sin la inversión de los elementos, falta ese ascenso tonal inicial: la oración ofrece un paulatino descenso melódico que llega hasta la última sílaba acentuada, después de la cual sigue, como en el caso anterior, el contorno melódico ascendente.

Ahora hace falta plantearse el problema de hasta qué punto se relacionan dichos cambios del contorno melódico con la inversión de los elementos en una pregunta absoluta y cuáles son las consecuencias de este fenómeno para la definición del melodema en español.

Brevemente, podemos decir que, con la inversión de los elementos, se LXVII, $10^{\circ}-2 .^{\circ}-3$ 
altera la coincidencia entre la sílaba proeminente de la oración y la última sílaba acentuada: $\mathrm{Si}$ el centro enunciativo de la oración llega al principio de la misma, se produce también cierto deslizamiento de la sílaba proeminente, el cual, como hemos señalado antes, tiene por consecuencia una mayor extensión de la forma melódica relevante y, por consiguiente, también ciertos lambios de su línea tonal.

Por lo tanto, somos de la opinión de que se trata de dos melodemas (y, por consiguiente, entonemas) distintos, ya que se diferencian no sólo por su contorno melódico, sino también por su posición entre los demás medios lingüísticos que sirven en español para la delimitación, diferenciación e integración contextual de una oración (enunciado), ya que cada uno de ellos corresponde a un distinto orden de los elementos en la pregunta absoluta.

Como resumen de nuestras reflexiones, podemos concluir que la pregunta absoluta en español posee dos melodemas sinónimos: uno ascendente pregunta por entonación y el otro descendente-ascendente pregunta por inversión, delimitados por la sílaba proeminente del centro entonativo de la oración.

3.2.3. Metapreguntas. Bajo el concepto de "metapreguntas" comprendemos aquellas oraciones interrogativas pronominales, incluidas generalmente dentro de las variantes afectivas o matices de cortesía, etc., destacándose el hecho de que el hablante parece saber la respuesta (cf. QUilis, FernánDEZ, 1973, pág. 177). Su contorno melódico también suele considerarse relevante a partir de la última sílaba acentuada:

I.

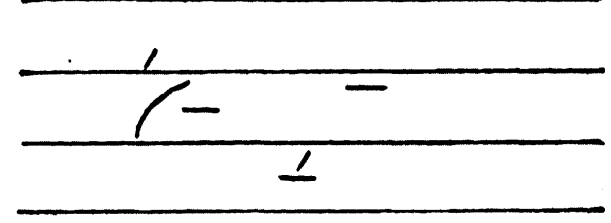

¿Cuánto debo?

Ja entonación de la pregunta pronominal en este caso ser,a paulatinamente descendente a partir de la s,laba proeminente de la oración, la cual coincide, en nuestro caso, con la sílaba acentuada del interrogativo. No obstante, en el contorno melódico de esta "metapregunta" aparece todavía un ascenso tonal a partir de la última sílaba acentuada, lo que lo asemeja al contorno melódico de una pregunta absoluta (cf. QUiLIs, FERNíndEZ, 1973, 
pág. 178). Por lo tanto, consideramos como fonológicamente relevante la línea tonal a partir de la mencionada sílaba acentuada del interrogativo correspondiente a la sílaba proeminente de la metapregunta y definimos su melodema como descendente-ascendente.

Al comparar el melodema de la metapregunta con el de la pregunta absoluta, vemos que existe una coincidencia en el contorno melódica de ésta y del de la pregunta absoluta con la inversión de los elementos.

Por lo tanto, cabe hacerse la pregunta siguiente: ¿se trata de un solo melodema empleado en dos tipos de preguntas, o se trata de dos melodemas homónimos?

Para poder resolver este problema, tenemos que ver la posición del contorno melódico en cuestión en la jerarquía comunicativa en español. En primer lugar, podemos decir que se emplea en las preguntas en las cuales no representa el único portador del carácter interrogativo de la oración: En las preguntas absolutas aparece ligado a la inversión de los elementos; las "metapreguntas" poseen el interrogativo que señala su carácter interrogativo. Este hecho podría tomarse por afinidad entre el aprovechamiento funcional de dicho melodema. No obstante, a pesar de esta afinidad, en la definición de la posición del contorno melódico descendente-ascendente hace falta, a nuestro parecer, tomar en cuenta los medios lingüísticos, mediante los cuales, aparte de la entonación, está marcado el valor interrogativo de las oraciones en cuestión.

Al concentrar nuestra atención en este último problema, vemos que aquí sí aparece una diferencia fundamental: la inversión de los elementos en la pregunta representa un medio sintáctico para expresar el valor interrogativo de la oración, mientras que los interrogativos, que tienen la misma función, representan un medio lexical. Es decir, el contorno melódico descendente ascendente en la pregunta absoluta con la inversión de los elementos en español está ligado a otros medios lingüísticos que el mismo contorno melódico en la metapregunta, y, por lo tanto, ocupa una posición diferente en la jerarquía comunicativa de cada una de las preguntas en cuestión.

Dadas las diferencias en la posición del contorno melódico mencionado en la jerarquía comunicativa según la oración en que aparece, consideramos indispensable hablar en este lugar de dos melodemas homónimos: Uno de la pregunta absoluta con la inversión de los elementos y el otro de la metapregunta, cada uno con su núlero y variedad de alómelos.

4. Partiendo del hecho de que los medios melódicos son los fundamentales, aunque no los únicos fenómenos entonológicos, hemos tratado de determinar el número de los melodemas esenciales en español. En los análisis realizados hasta ahora hemos dejado aparte los tipos específicos de expresiones como, 
por ejemplo, las enumeraciones, las preguntas disyuntivas, etc., para determinar, ante todo, las características básicas de la melodía oracional en español. Por lo tanto, hemos dedicado nuestra atención ante todo a las simples oraciones afirmativas e interrogativas, en las cuales hemos detectado hasta ahora tres contornos melódicos relevantes:

1. Contorno melódico descendente (en las oraciones afirmativas y en las preguntas pronominales).

2. ${ }^{\circ}$ Contorno melódico ascendente (en las preguntas absolutas sin inversión de los elementos).

3. Contorno melódico descendente-ascendente (en las preguntas absolutas con inversión de los elementos, en las "metapreguntas").

Como ya se dijo antes, el valor lingüístico de los medios entonológicos no se puede determinar sin tomar en cuenta su posición en la jerarquía comunicativa en la lengua determinada. Desde este punto de vista, parece indiscutible sólo la existencia de un melodema ascendente, identificado con el contorno melódico relevante de las preguntas absolutas sin inversión de los elementos.

Al tratar de comparar la posición del contorno melódico relevante en la jerarquía comunicativa en las oraciones afirmativas y en las preguntas pronominales, se nota que mientras en las primeras es el único portador del carácter afirmativo de la oración, en las segundas se relaciona con el interrogativo que en este tipo de oraciones determina su valor no-afirmativo. Por lo tanto, nos parece preferible hablar de dos melodemas descendentes homónimos: uno de la oración afirmativa y el otro de la pregunta pronominal.

Al analizar desde este punto de vista el tercer contorno melódico relevante, el descendente-ascendente, vemos un fenómeno específico: en cada uno de los dos tipos de oraciones, el melodema no es el único medio portador de su valor interrogativo. En este tipo de preguntas absolutas, el valor interrogativo (mejor dicho, el no-afirmativo) es apoyado por la inversión de los elementos, en las "metapreguntas" pronominales es portado por el interrogativo. Dadas las reglas del orden de los elementos en la oración española, se nota ya de la formulación misma de las relaciones entre el contorno melódico y otros medios lingüísticos para definir, delimitar e integrar contextualmente las oraciones hispánicas, que un mismo contorno melódico descendente-ascendente ocupa diferentes posiciones en la jerarquía comunicativa, en la pregunta absoluta con inversión de los elementos y en la de la "metapregunta" pronominal. Por lo tanto, también en este caso nos parece justificado hablar de dos melodemas descendentes-ascendentes homónimos: 
uno de la pregunta absoluta con la inversión de los elementos, y el otro en la "metapregunta" pronominal.

En nuestros análisis hemos encontrado, pues, cinco melodemas: uno ascendente, dos descendentes y dos descendentes-ascendentes, diferenciados por su posición en la jerarquía conunicativa en español. Hemos tomado en cuenta cuatro tipos diferentes de oraciones, y de eso se desprende otro fenómeno que ofrecen los mismos medios entonológicos en español : la sinonimia. En nuestros análisis, hemos encontrado, hasta ahora, un solo caso de sinonimia de los melodemas (haciendo abstracción de las variantes dialectales): se trata de los contornos melódicos relevantes de la pregunta absoluta: es el melodema ascendente y el descendente-ascendente, ligado a la inversión de los elementos en este tipo de la oración interrogativa. También en este caso los melodemas, a pesar de ser sinónimos, se diferencian, naturalmente, por su posición en la jerarquía comunicativa en español.

Como ya hemos reiterado varias veces, no consideramos agotados nuestros análisis ni nuestras conclusiones: en primer lugar, no son universales: no abarcan todos los tipos de oraciones y enunciados en español; y en segundo lugar, es lógico que una concepción de cierto fenómeno lingüístico no puede ser universal, si no se ha aspirado a ello. Sólo hemos querido presentar nuestro enfoque de un importante fenómeno lingüístico tan controvertido como es la melodía oracional, e introduciendo el concepto de la jerarquía comunicativa, determinar su posición entre los demás medios lingüísticos que en español sirven para delimitar, estructurar e integrar contextualmente una oración (un enunciado).

JanA KulLová.

\section{BIBLIOGRAFIA}

Alarcos Llorach, E. (1964): Fonología española, Madrid, Gredos.

Antipova, A. M. (1979): "Interrelation of Rhytm and Other Components of Intonation" (Abstract), Proceedings of the $9^{\text {th }}$ International Congress of Phonetic Sciences I, Copenhague, pág. 362.

Cárdenas, D. N. (1960): Introducción a una comparación fonológica del español y del inglés, Washington, D. C.

Ceplrtis, L. K. (1974) : Analis rechevoi intonatsii (Análisis de la entonación del habla), Riga.

Dalbor, J. B. (1969) : Spanish Pronuntiation: Theory and Practice, New York.

Daneš, F. (1967): "Order of Elements and Sentence Intonation", To Honor Roman Jakobson, La Haya, págs. 499-512.

Gili Gays, S. (1970): Curso superior de sintaxis española, Barcelona, Bibliograf, S. A.

Guiraud, P. (1974) : La semiología, Buenos Aires.

Hocketт, Сн. (1972): Curso de lingüistica moderna, Buenos Aires. 
Martinet, A. (1972) : Fonología como fonética funcional, Buenos Aires.

Matluck, J. M. (1965) : Entonación hispánica, México, D. F.

Navarro T., T. (19661) : Manual de entonación española, México, D. F. (1966²): Manual de pronunciación española, La Habana.

Quilıs, A. (1971): "Caracterización fonética del acento español", Travaux de Linguistique et Littérature, IX, I., Strasbourg, págs. 53-72.

(1981) : Fonética acústica de la lengua española, Madrid, Gredos.

Quilis, A., y Fernández, J. A. (1973): Curso de fonética y fonología españolas, Madrid.

Romportu, M. (1961): "Zum Wesen der Intonation", Proceedings of the $4^{\text {th }}$ Congress of Phonetic Sciences, pág. 749.

(1970): "On the Phonic Analysis of Language", AUC-Phonetica Pragensia II, Praga, págs. 9-18.

(1971): "K synonymii a homonymii intonačních prostředků" (Acerca de la sinonimia y homonimia de los medios entonativos), AUC-Slavica Pragensia, XII, Praga, págs. 209-217.

(1973) : Studies in Phonetics, Praga-La Haya, París.

(1981): Kadence, intonem, melodem (Cadencia, entonema, melodema), Conferencia en la Facultad de Filosofía y Letras.

TICHÝ, O. (1961): "Recursos de incorporación contextual de la oración", $A U C-R o-$ manistica Pregensia II, Praga, págs. 91-97. 